\title{
Developing high throughput quantitative PCR assays for diagnosing Ikeda and other Theileria orientalis types common to New Zealand in bovine blood samples
}

\author{
DJ Pulford *\$ , E Gias*, IM Bueno*, AMJ McFadden* \\ * Ministry for Primary Industries, Investigation and Diagnostic Centre, 66 Ward St, Wallaceville, Upper Hutt, New Zealand \\ $\checkmark$ Author for correspondence. Email: David.Pulford@mpi.govt.nz
}

Supplementary Figure 1. Mapping PCR oligonucleotide primers and probes designed for quantitiative PCR to a CLUSTALW multiple alignment of major piroplasm surface protein nucleotide sequences from GenBank or collected from New Zealand samples for Theileria orientalis Ikeda, Chitose and Buffeli types.

Genotype consensus sequences for New Zealand field samples were generated from raw MPSP (Ota et al. 2011) amplicon nucleotide sequences if they had no ambiguous base calls in the raw data, and they were positive by conventional PCR to only one T. orientalis type. Consensus sequences for Ikeda (NZ Ikeda Con) were generated from KP795979, KP795981, KP795982, for Buffeli (NZ Buffeli Con) from KP795978 and KP795983 using CLUSTALW, and for Chitose (NZ Chitose Con) KP795977 (Pulford et al. 2015). A multiple-alignment of New Zealand type consensus sequences with reference sequences for type 1 Chitose (THETS32KC), type 2 lkeda (THEPMIPI) and type 3 Buffeli (THEPMIPW) was generated with CLUSTALW. Nucleotide positions shared with the consensus sequence are shown as dots, otherwise variations from the consensus are shown with a single letter code polymorphism. Primer and probe positions are shown as arrows, Th.ori primers are shown in green and grey, type specific qPCR primers are shown in shades of green, and probes in red.

Ota N, Mizuno D, Kuboki N, Igarashi I, Nakamura Y, Yamashina H, Hanzaike T, Fujii K, Onoe S, Hata H et al. Epidemiological survey of Theileria orientalis infection in grazing cattle in the eastern part of Hokkaido, Japan. Journal of Veterinary Medical Science 71, 937-44, 2009

Pulford DJ, McFadden, AMJ, Hamilton, JS, Donald J. Investigation of the index case herd and identification of the strain types of Theileria orientalis associated with outbreaks of bovine anaemia in New Zealand in 2012. New Zealand Veterinary Journal doi: 10.1080/00480169.2015.1090355, 2015

The content of this supplementary information has not been edited. All risk and liability rest with the authors. 


\section{Supplementary Information}

Consensus NZ Ikeda Con

THEPMIPI

NZ Chitose Con

THETS32KC

NZ Buffeli Con

THEPMIPW

Consensus NZ Ikeda Con

THEPMIPI

NZ Chitose Con

THETS32KC

NZ Buffeli Con

THEPMIPW

Consensus

THEPMIPI

NZ Chitose Con

THETS32KC

NZ Buffeli Con

THEPMIPW

Consensus NZ Ikeda Con

THEPMIPI

NZ Chitose Con

THETS32KC

NZ Buffeli Con

THEPMIPW

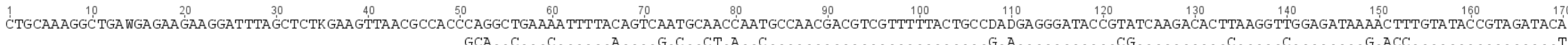
GCA..C...C...............CT.A..C.

......A............G .........................ACC....

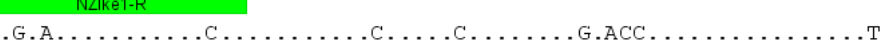
А........................

akent-

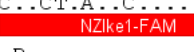

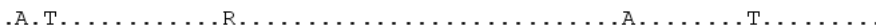

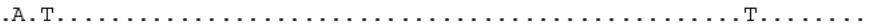

$\ldots$

.TCG..T..... T... T...... T.

.......

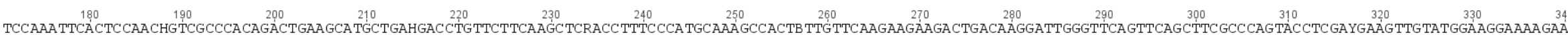

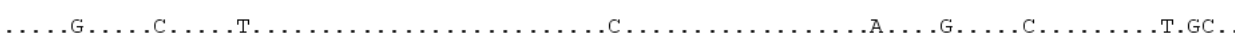

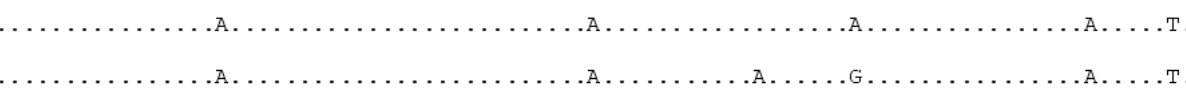

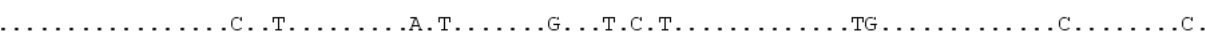

. TG. . . .

........T..C...T.

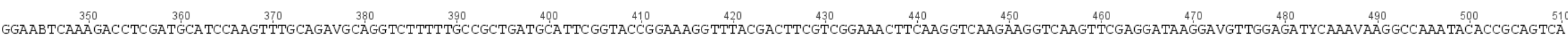

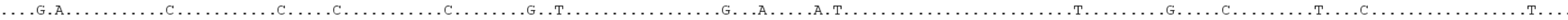

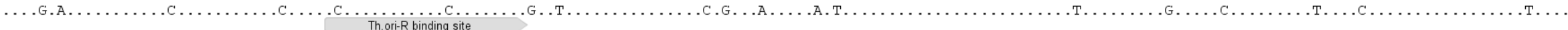

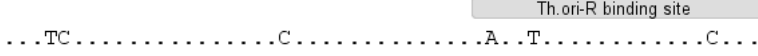

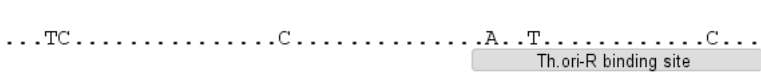

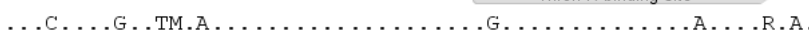

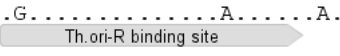

T. . T..............
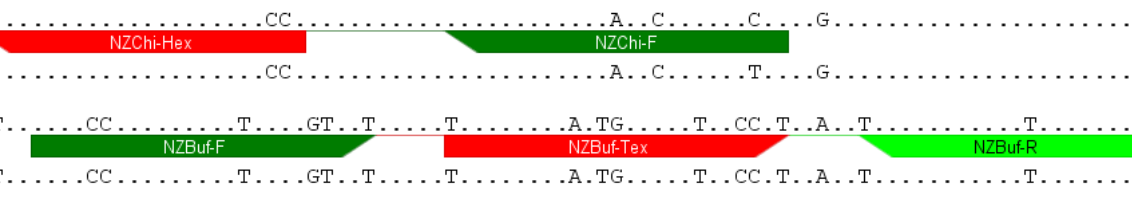

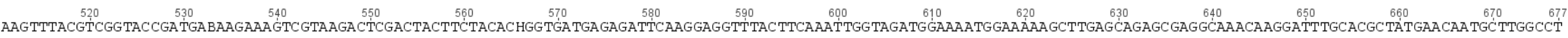

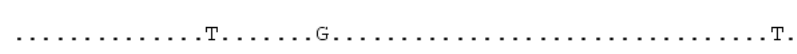


Supplementary Figure 2. The copy number of Theileria orientalis lkeda genomes in blood measured using an Ikeda-specific quantitative PCR. Samples shown were obtained from a dairy herd in Ruawai, Northland from cattle with clinical signs of infection with $T$. orientalis.

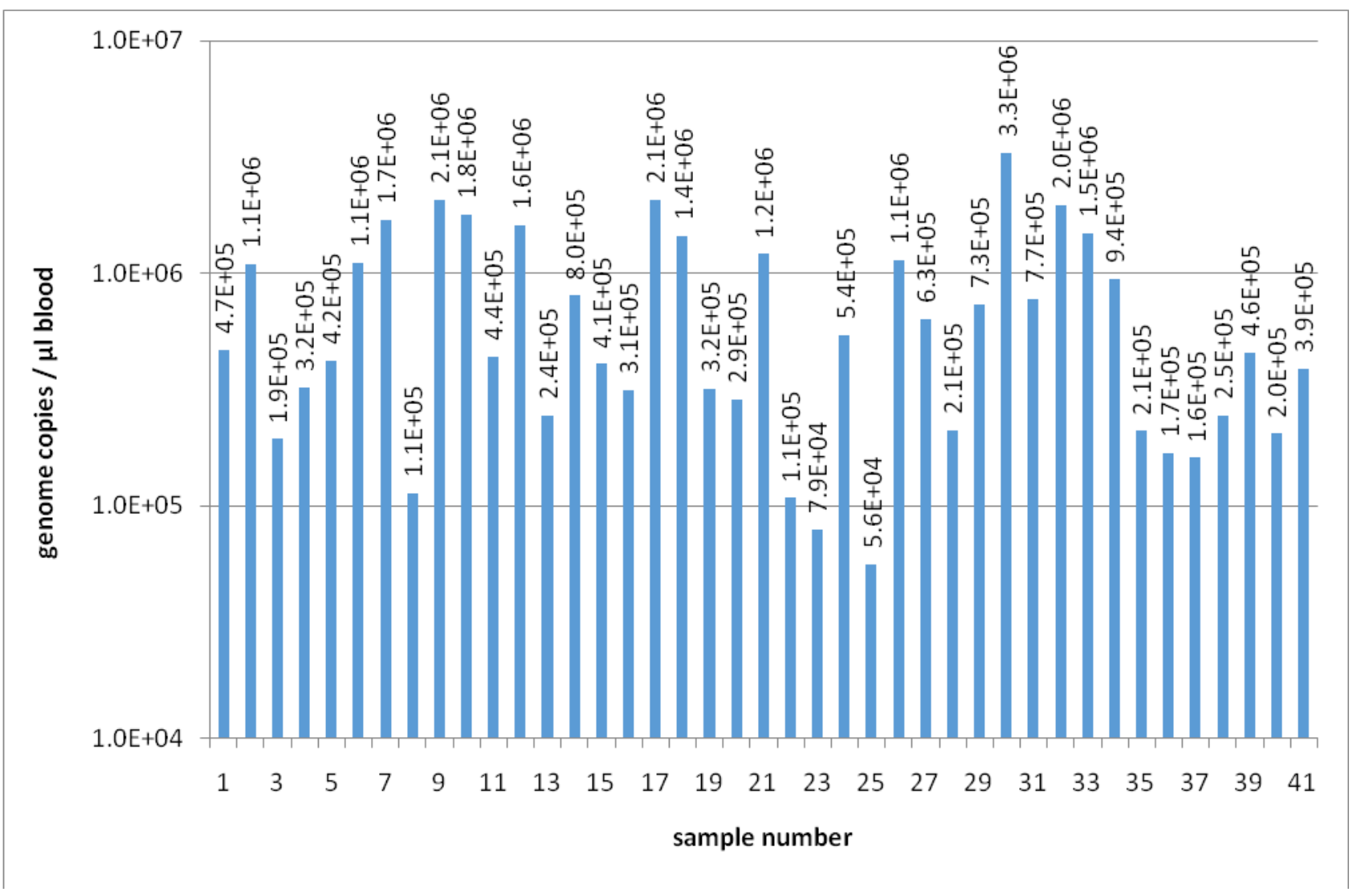

